\title{
Hernia de Spiegel y apendicitis aguda necrosada. Un reporte de caso
}

\author{
Spiegelian hernia and necrotic acute appendicitis. Case report
}

Alejandro Martín-Mattar*, Rigoberto Isarraraz-Flores y Juan P. Ávila

Servicio de Cirugía General, Hospital General de Mexicali, Mexicali, Baja California, México

\section{Resumen}

Antecedentes: La hernia de Spiegel es un tipo de hernia ventral poco frecuente que ocurre cuando existe un defecto en la línea semilunar (línea de Spiegel), a través del cual hay protrusión de grasa preperitoneal o un saco peritoneal con o sin una víscera intraabdominal. Caso clínico: Mujer de 52 años que ingresa por dolor abdominal y un abultamiento en el cuadrante inferior derecho de 15 centímetros. En quirófano se realiza plastia de pared y al abrir el saco herniario se identifica apendicitis necrosada, por lo que se realiza apendicectomía tipo Pouchet y plastia de pared con malla de polipropileno. Conclusión: La incidencia de la hernia se Spiegel es muy baja y lo es aún más cuando se asocia a apendicitis aguda. Dada su baja frecuencia, es importante la sospecha clínica. El tratamiento es quirúrgico.

Palabras clave: Hernia de Spiegel. Apendicitis. Plastia. Apendicectomía.

\begin{abstract}
Background: Spiegel hernia is a rare type of ventral hernia that occurs when there is a defect in the semilunar line (Spiegel line) through which there is protrusion of preperitoneal fat or a peritoneal sac with or without an intra-abdominal viscus. Clinical case: A 52-year-old female patient admitted for abdominal pain and tumor in the lower right quadrant of $15 \mathrm{~cm}$. In the operating room, wall plasty was performed and when hernia sac was opened, necrotic appendicitis was identified. Conclusion: The incidence of hernia is Spiegel is very low and is lower when associated with acute appendicitis. Given its low frequency, clinical suspicion is important. The treatment is surgical.
\end{abstract}

Key words: Spiegelian hernia. Appendicitis. Plasty. Appendectomy.

\section{Introducción}

La hernia de Spiegel es un tipo de hernia ventral poco frecuente que ocurre cuando existe un defecto en la línea semilunar (línea de Spiegel), a través del cual hay protrusión de grasa preperitoneal o un saco peritoneal con o sin una víscera intraabdominal.
Puede ser de carácter congénito o adquirido, y su tratamiento es quirúrgico, teniendo la alternativa de cirugía convencional (abordaje abierto) o abordaje laparoscópico. La hernia de Spiegel supone el 0.12$2 \%$ de las hernias de la pared abdominal y se presenta en todas la edades, con un pico de incidencia entre los 50 y 60 años ${ }^{1}$.

\section{Correspondencia:}

*Alejandro Martín-Mattar

Vazco de Quiroga, 640

Col. ProHogar

Fecha de recepción: 13-02-2019

C.P. 21240 , Mexicali, B.C., México

E-mail: amartin_mattar@hotmail.com
Fecha de aceptación: 08-01-2020

DOI: $10.24875 / C I R U .20001112$
Cir Cir. 2020;88(6):782-786

Contents available at PubMed www.cirugiaycirujanos.com 0009-7411/@ 2020 Academia Mexicana de Cirugía. Publicado por Permanyer. Este es un artículo open access bajo la licencia CC BY-NC-ND (http://creativecommons.org/licenses/by-nc-nd/4.0/). 
La mayoría de las hernias de Spiegel ocurren en la parte inferior del abdomen, donde se encuentra la vaina posterior deficiente. También se la denomina hernia ventrolateral espontánea o hernia de línea semilunar. El anillo de la hernia es un defecto bien definido en la aponeurosis del músculo transverso. El diagnóstico de hernia de Spiegel es difícil. La hernia puede ser interparietal sin masa obvia en la inspección o la palpación. La hernia de Spiegel ha sido reparada por abordajes tanto convencionales como laparoscópicos. La mayoría de las veces, cuando se ha utilizado la laparoscopia como modalidad de tratamiento para la hernia de Spiegel, se ha realizado por abordaje transabdominal 0 extraperitoneal².

\section{Caso clínico}

Mujer de 52 años que acude al servicio de urgencias por presentar un cuadro de dolor abdominal en el cuadrante inferior derecho de 18 horas de evolución. Como antecedentes de importancia cuenta con hipertensión arterial e hipercolesterolemia de 1 año de diagnóstico, en tratamiento con enalapril y bezafibrato; colecistectomía hace 2 años; gestaciones 2 y cesáreas 2. Niega antecedentes alérgicos, traumáticos, transfusionales, infecto-contagiosos, etilismo, tabaquismo y toxicomanías. Originaria de Mexicali, Baja California, ama de casa, sedentaria, su dieta se basa en alto consumo de harinas refinadas, azúcares, grasas, pollo tres veces a la semana, carne roja una vez a la semana y escasa ingesta de frutas y verduras. Inicia el padecimiento actual 18 horas antes de su ingreso al servicio de urgencias con dolor de inicio agudo en el cuadrante inferior derecho, asociado a náuseas, sin llegar al vómito, sin irradiación ni migración, y sin otra sintomatología agregada. A la exploración se observa protrusión palpable en el cuadrante inferior derecho de consistencia pétrea, levemente móvil, dolorosa a la palpación, de aproximadamente $15 \mathrm{~cm}$ de diámetro. Se realiza la evaluación de la escala de Ripasa y reporta 9.5, y un Alvarado de 6 puntos.

Ante el dolor abdominal y la presencia de tumor, se decide realizar tomografía simple de abdomen, 1 hora después de su ingreso a urgencias, previa colocación de soluciones parenterales, analgesia y toma de muestras de sangre (Tabla 1). En el estudio se encuentra un defecto herniario de unos $4.5 \mathrm{~cm}$ localizado entre el borde lateral del músculo recto derecho del abdomen y la línea semilunar homolateral, por

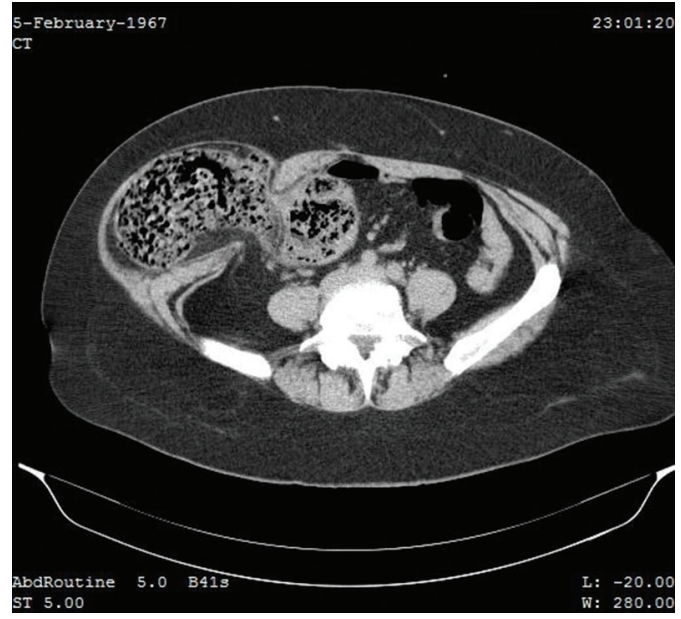

Figura 1. Corte transversal en el que se evidencia el defecto herniario en la línea de Spiegel.

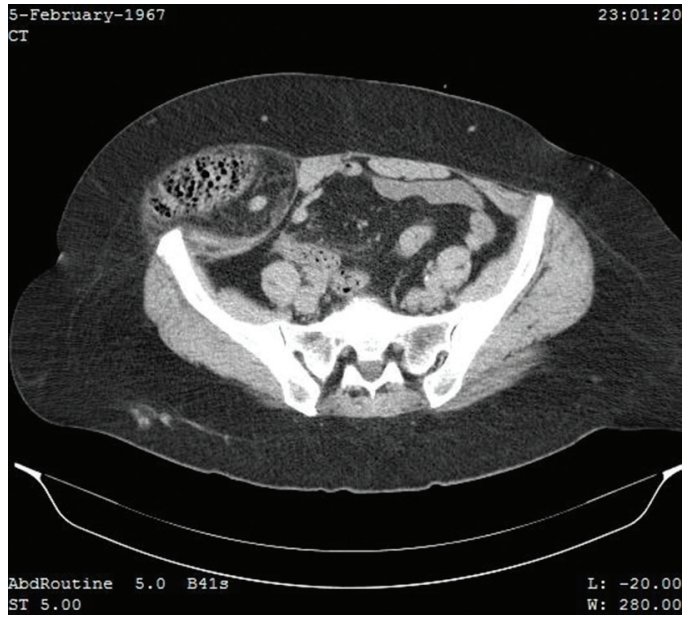

Figura 2. Corte transversal en el que se evidencia el saco herniario con contenido de colon ascendente y ciego.

donde protruye un saco que respeta la aponeurosis del oblicuo menor y que contiene una pequeña porción del ileon, ciego y colon ascendente, con abundante material de residuo en su interior, además de grasa de la cavidad peritoneal, con mínimos cambios inflamatorios circundantes al orificio herniario. De forma incidental también se aprecian múltiples saculaciones menores de $0.5 \mathrm{~cm}$ a lo largo de la pared del colon descendente (Figs. 1-4).

Media hora después del reporte por parte del servicio de radiologia e imagen se decidió realizar tratamiento quirúrgico con plastia abdominal y malla, previa profilaxis antibiótica con ceftriaxona $(1 \mathrm{~g} / 12 \mathrm{~h}$ por vía intravenosa [i.v.]), ketorolaco (30 mg/8 h i.v.), metamizol (1 g/8 h i.v.) y omeprazol (40 mg/24 h i.v.), 


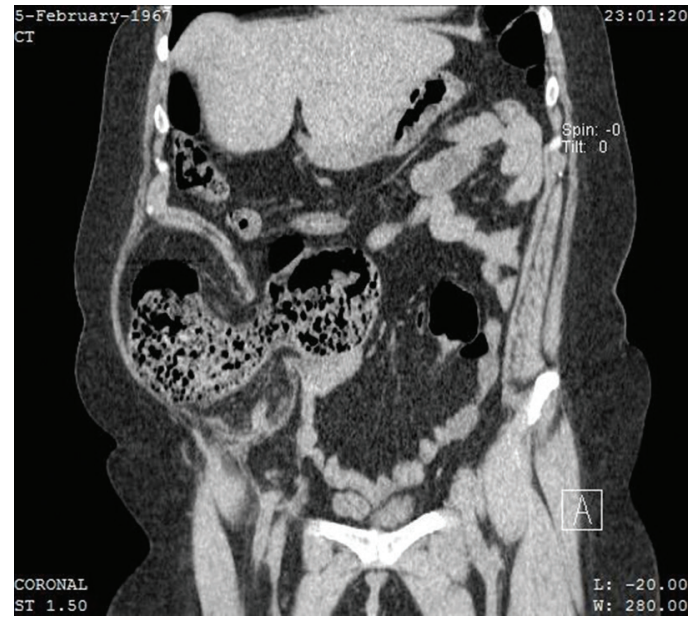

Figura 3. Corte coronal en el que se evidencia la hernia de Spiegel.

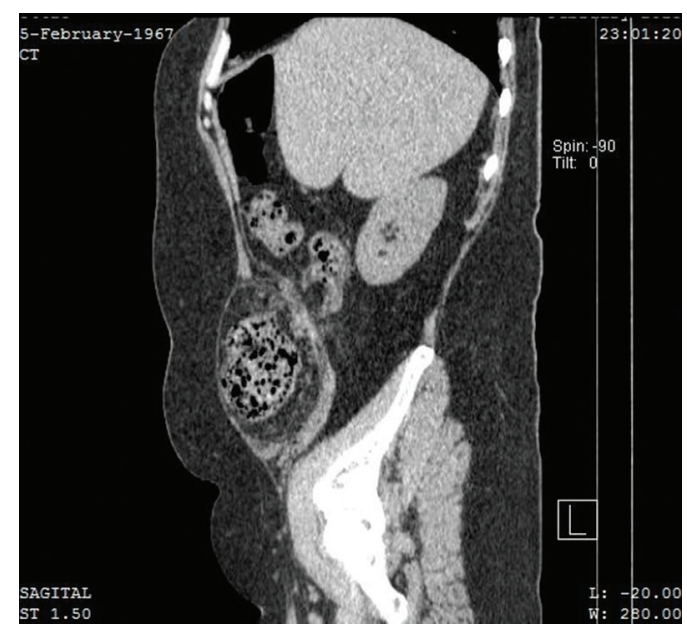

Figura 4. Corte sagital de abdomen.

todos iniciados al momento del diagnóstico y de la decisión quirúrgica, hasta 7 días después de su egreso hasta completar así 10 días de tratamiento. Una vez en sala de quirófano, previa colocación de bloqueo subdural con bupivacaína pesada (15 mg), asepsia y colocación de campos estériles, se realiza incisión transversa por encima de la hernia, se diseca por planos hasta encontrar la fascia del oblicuo mayor abombada, se realiza incisión con bisturí y se encuentra la línea de Spiegel con un defecto de 5 $\mathrm{cm}$ y un saco herniario de $15 \mathrm{~cm}$. Se abre el saco y se evidencia el apéndice retrocecal de $9 \times 0.5 \mathrm{~cm}$ en fase necrosada en sus dos tercios distales (Fig. 5), por lo que se realiza apendicectomía tipo Pouchet con Vicryl ${ }^{\circledR} 2-0$, se explora la cavidad y no se evidencia ninguna otra lesión intraabdominal. Se

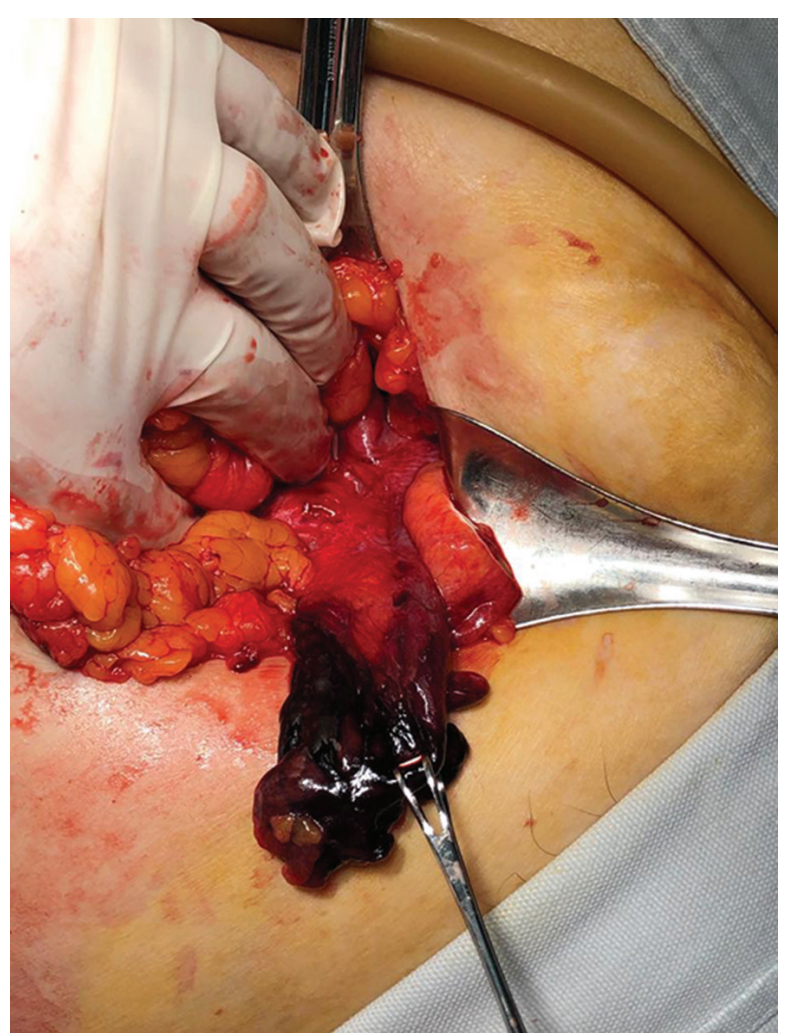

Figura 5. Hernia de Spiegel con saco herniario abierto y apendicitis necrosada en su interior.

realiza plastia de pared afrontando la línea de Spiegel (recto anterior derecho y transverso) y posteriormente se cierra fascia del oblicuo mayor con polipropileno (Prolene ${ }^{\circledR}$ ) del 1, se diseca aponeurosis circundante sana, se coloca una malla de Prolene ${ }^{\circledR}$ de $10 \times 10 \mathrm{~cm}$ y se fija con polipropileno $2-0$. Se coloca un drenaje cerrado en el tejido celular subcutáneo, se afronta este y se cierra la piel con punto medio Sarnoff.

La paciente presenta una evolución favorable, signos vitales dentro de los parámetros normales, tolera la vía oral y el gasto por el drenaje es serohemático escaso (unos $30 \mathrm{ml}$ ). Se decide el egreso al tercer día de posoperatorio para impregnación antibiótica y se da de alta con doble esquema de antibiótico a base de ciprofloxacino $(500 \mathrm{mg} / 12 \mathrm{~h}$ por vía oral [v.o.] y metronidazol (500 mg/8 h v.o.), y paracetamol (500 mg/8 h v.o.). Se citó para retiro del drenaje a los 5 días de posoperatorio, evidenciando un gasto seroso escaso (unos $20 \mathrm{ml}$ en 24 horas), por lo que se retira. Posteriormente, 15 días después del retiro de drenaje, fue valorada en consulta externa, encontrando la herida quirúrgica ya 
Tabla 1. Exámenes de laboratorio al ingreso (biometría hemática, química sanguínea y tiempos de coagulación)

\begin{tabular}{lc}
\hline Parámetro & Resultado \\
\hline Hemoglobina & $16 \mathrm{mg} / \mathrm{dl}$ \\
Hematocrito & $50.2 \%$ \\
Leucocitos & $16.0010^{\star} 3$ \\
Neutrófilos & $86 \%$ \\
Plaquetas & $29510^{*} 3$ \\
Glucosa & $85 \mathrm{mg} / \mathrm{dl}$ \\
Urea & $34 \mathrm{mg} / \mathrm{dl}$ \\
Creatinina & $1.3 \mathrm{mg} / \mathrm{dl}$ \\
Tiempo de protrombina & $17 \mathrm{~s}$ \\
INR & 1.05 \\
Tiempo de tromboplastina parcial & $23.5 \mathrm{~s}$ \\
\hline INR: International Normalized Ratio.
\end{tabular}

cicatrizada, sin defectos herniarios y sin datos de alarma abdominal.

\section{Discusión}

Las hernias de Spiegel, también conocidas como hernias ventrales laterales o hernias de la línea semilunar (Fig. 1), se consideran una afección rara, ya que constituyen el $0.12-2 \%$ de todas las hernias de la pared abdominal. Adriaan van der Spiegel (1578-1625), anatomista de Bélgica, fue el primero en describir la línea semilunar. Posteriormente, Joseph Klinkosch describió por primera vez este tipo de hernia como un defecto en la línea semilunar, acuñando el término de «hernia de Spiegel» ${ }^{3}$.

Se han considerado varios factores que pudieran contribuir a su etiología, como un aumento de la presión intraabdominal, un deterioro de la pared abdominal, alteraciones del colágeno, la edad, la obesidad, los embarazos múltiples, la pérdida de peso, la enfermedad pulmonar obstructiva crónica y los traumatismos ${ }^{4}$.

La hernia de Spiegel es un tipo raro de hernia abdominal y el apéndice muy pocas veces se encuentra en el contenido herniario; según los informes, se han visto diversos órganos intraabdominales en dicha hernia, pero la presencia del apéndice es excepcional $^{6}$. Los síntomas iniciales son generalmente inespecíficos, lo que retrasa el correcto diagnóstico y la instauración del tratamiento adecuado. Los estudios de imagen son en particular útiles para el diagnóstico ${ }^{5}$.

El tratamiento es quirúrgico y existen varios abordajes. La reparación se ha descrito utilizando distintas técnicas, desde cierre primario hasta reparación con malla. El cierre primario se considera una reparación con tensión y cursa con un alto porcentaje de recidivas (4\%). La reparación con malla (sin tensión) se asocia con una disminución significativa de las recidivas (0-0.7\%). La desventaja de estas técnicas es que requieren una incisión y una disección amplias, por lo que cursan con dolor posoperatorio importante y requieren hospitalización ${ }^{7}$.

\section{Conclusión}

La presencia de una hernia de Spiegel asociada con apendicitis aguda es infrecuente y se ha descrito muy pocas veces en la literatura médica. Dada su baja frecuencia, es importante la sospecha y sobre todo la correlación clínica y paraclínica para llegar a un diagnóstico oportuno. El tratamiento es quirúrgico en todos los casos 2 .

La importancia del caso clínico que presentamos se debe a la rareza de este padecimiento, ya que se presenta en menos del $2 \%$ de la poblacion general y cuando se asocia a apendicitis aguda el porcentaje es aún menor. El dolor abdominal es un motivo frecuente por el que los pacientes acuden a urgencias y razón de interconsulta al servicio de cirugía. La clínica de la hernia de Spiegel puede confundirse con otras afecciones más frecuentes y por ello toma importancia una valoracion integral, considerar los diagnósticos diferenciales y no dejar de lado otras posibilidades etiológicas no tan frecuentes.

\section{Agradecimientos}

Los autores agradecen al Hospital General de Mexicali, al servicio de imagenología y al servicio de cirugía general el apoyo para la realización de este trabajo. Al Dr. Juan Pablo Ávila, por ser el asesor de este caso clínico, y al personal de quirófano, internos y residentes de anestesiología. Al personal de enfermería, que tuvo un papel importante en el proceso diagnóstico y terapéutico del paciente.

\section{Financiamiento}

Los autores declaran que no se recibió ningún patrocinio para la realización de este trabajo. 


\section{Conflicto de intereses}

Los autores declaran que no se generó ningún conflicto de intereses, ya que no hubo relaciones personales ni financieras que puedan influenciar de manera inapropiada las acciones tomadas en el momento de la realización de este trabajo.

\section{Responsabilidades éticas}

Protección de personas y animales. Los autores declaran que para esta investigación no se han realizado experimentos en seres humanos ni en animales.

Confidencialidad de los datos. Los autores declaran que han seguido los protocolos de su centro de trabajo sobre la publicación de datos de pacientes.

Derecho a la privacidad y consentimiento informado. Los autores han obtenido el consentimiento informado de los pacientes y/o sujetos referidos en el artículo. Este documento obra en poder del autor de correspondencia.

\section{Bibliografía}

1. Cox MJ, Adiamah A, Chowdhury A, Shah A. Rare and unusual case of perforated appendicitis in a Spigelian hernia. BMJ Case Rep. 2017;2017:bcr2017221851.

2. Mittal T, Kumar V, Khullar R, Sharma A, Soni V, Baijal M, et al. Diagnosis and management of spigelian hernia: a review of literature and our experience. J Minim Access Surg. 2008;4:95-8.

3. Zaki W, Alawad AAM. Spigelian Hernia: a rare case report. Ann Clin Case Rep. 2017;2:1288.

4. Thomas MP, Avula SK, England R, Stevenson L. Appendicitis in a Spigelian hernia: an unusual cause for a tender right iliac fossa mass. Ann R Coll Surg Engl. 2013;95:e66-8.

5. Kaliszewski K, Kozakiewicz M, Lubieniecka B, Bronowicki J, Szelachowski P, Tabola R, et al. Spigelian hernia containing an incarcerated appendix - a case report and literature review. J Clin Case Rep. 2017;7:1057.

6. Reinke C, Resnick A. Incarcerated appendix in a Spigelian hernia. J Surg Case Rep. 2010;2010:3.

7. Murillo-Zolezzi A, Hernánez-López A, Puente-Espel J. Hernia de Spiegel, presentación de un caso y revisión de la literatura. Rev Mex Cir Endoscop. 2011;12:130-4. 